\title{
Micropulse trans-scleral \\ cyclophotocoagulation: a light before the end of the tunnel
}

\section{Liza-Sharmini Ahmad Tajudin}

Department of Ophthalmology, School of Medical Sciences, Universiti Sains Malaysia Health Campus, Kota Bharu, Kelantan, Malaysia

*This article contains an erratum to the original.

Refractory glaucoma is defined as uncontrolled glaucoma with evidence of progression of the disease structurally and functionally despite maximum tolerable medical therapy, surgical therapy, or combination of medical and surgical therapy and high risk of trabeculectomy failure. ${ }^{1}$ Based on the definition, management of refractory glaucoma is challenging, even more so in those with end-stage glaucoma; there is desperation in both patients and ophthalmologists.

Surgical management, and especially glaucoma drainage device (GDD) implantation, may provide better intraocular pressure (IOP) control with presumed better chances of preventing further progression in refractory glaucoma. ${ }^{2}$ However, the outcome of GDD implantation is not as expected, with a rate of failure of $10 \%$ per year. ${ }^{2}$ Trans-scleral cyclophotocoagulation (TSCPC) laser therapy with continuous wave $(\mathrm{CW})$ to reduce aqueous production through destruction of ciliary body has regained popularity. ${ }^{3}$ Unfortunately, the higher undesirable ocular complications and pain during the procedure have diminished its popularity.

Recent technological advances have brought micropulse wave TSCPC, known as micropulse cyclophotocoagulation (MPCPC), to expand the surgical options for refractry glaucoma. ${ }^{4}$ In this issue of the Malaysian Journal of Ophthalmology, Chung et. al illustrate the potential effectiveness of MPCPC as adjunctive therapy in the Malaysian population. ${ }^{5}$ Based on their findings, MPCPC has shown good IOP reduction (IOP $<21 \mathrm{mmHg}$ or $20 \%$ reduction from baseline) in patients with refractory glaucoma who were not keen or suitable for surgical intervention. This retrospective review was conducted on a small group of patients (34 eyes of 24 patients) for a short period of time (6 months).

Similar findings have been observed in other populations., ${ }^{3,46-8}$ Most of the studies were retrospective in nature, and therefore subject to bias, especially due to inadequate documentation. . $3,4,7^{3}$ Comparison is not possible due to a lack of standardized definition of success (IOP reduction) for this laser procedure. The short 
follow-up period is another limitation in the majority of MPCPC reports, the longest follow-up being 18 months. The only consistent finding is that the procedure is less painful compared to TSCPC. However, no standardized scores for pain were used in the previous literature. Chung et al. applied a numerical rating scale to score the pain during MPCPC. ${ }^{5}$ In the future, a prospective, multicentre, randomized controlled trial with at least a 5-year follow-up period is recommended to evaluate this novel procedure.

With a single-use probe, MPCPC is quite costly, which is a major drawback. The tip of the probe (Micropulse P3 ${ }^{\mathrm{TM}}$, Iridex Corp., Mountain View, CA, USA) is quite large for small eyes such as Asian eyes. It may be a challenge in eyes with primary angle closure and small interpalpebral fissure. Subliminal TSCPC (SubCyclo ${ }^{\circledR}$, Quantel Medical, Cournon-d'Auvergne, France), uses the same principle of micropulse wave but with a smaller and reusable probe. ${ }^{9}$ Asians have highly pigmented eyes that may require less power delivery of $M P C P C$ compared to less pigmented individuals. The power settings for patients with good central vision may differ from painful, blind eyes. Thus, individualised power settings are recommended. Despite the limitations and lack of strong evidence, MPCPC has the potential of shining a light before the end of the tunnel: a gleaming hope for patients at the end-stage of glaucoma.

\section{*Erratum}

The author of this article wishes to amend the following sentence in the original editorial: "Subliminal TSCPC (SubCyclo ${ }^{\circledR}$, Quantel Medical, Cournon-d'Auvergne, France), uses the same principle of micropulse wave but with a smaller and reusable probe." "The sentence should read: "Subliminal TSCPC (SubCyclo ${ }^{\circledR}$, Quantel Medical, Cournob-d'Auvergne, France), uses the same principle of micropulse wave with smaller probe, but reusability of the probe is still not recommended by the manufacturer. ${ }^{9 "}$ Dr. Liza-Sharmini regrets the error.

\section{References}

1. Kitazawa Y, Yamamoto T, Sawada A, Hagiwara Y. Surgery for refractory glaucoma. Aust N Z J Ophthalmol. 1996; 24(4):327-332.

2. Patel S, Pasquale RL. Glaucoma Drainage Devices: A Review of the Past, Present, and Future. Semin Ophthalmol. 2010;25(5-6):265-270. doi: 10.3109/08820538.2010.518840

3. Aquino CM, Barton K, Tan AM, Sng C, Li X, Loon SC, Chew PT. Micropulse versus continuous wave transscleral diode cyclophotocoagulation in refractory glaucoma: a randomized exploratory study. Clin Exp Ophthal. 2015;43(1):40-46. doi: 10.1111/ceo.12360 
4. Tan AM, Chockalingam M, Aquino MC, Lim ZIL, See JLS, Chew PTK. Micropulse transscleral diode laser cyclophotocoagulation in the treatment of refractory glaucoma. Clin Exp Ophthalmol. 2010;38:266272. doi: 10.1111/j.1442-9071.2010.02238.x

5. Liu CC, Pappanayakan G, Ch'ng TW, et al. Micropulse trans-scleral cyclophotocoagulation: treatment outcomes of refractory glaucoma in Malaysia. Malaysian Journal of Opthalmology. 2020;2(4):258268.

6. Preda MA, Karancsi LO, Munteanu M, Stanca TH. Clinical outcomes of micropulse transscleral cyclophotocoagulation in refractory glaucoma: 18 months follow up. Lasers Med Sci. 2020;35:1487-1491.

7. Varikuti V, Shah P, Rai O, et al. Outcomes of Micropulse Transscleral Cyclophotocoagulation in Eyes With Good Central Vision. J Glaucoma. 2019;28(10):901-905. doi: 10.1097/IJG.0000000000001339

8. Zaarour K, Abdelmassih Y, Arej N, Cherfan G, Tomey K, Khoueir Z. Outcomes of Micropulse Transscleral Cyclophotocoagulation in Uncontrolled Glaucoma Patients. J Glaucoma. 2019;28(3):270-275. doi: 10.1097/IJG.0000000000001174

9. Lutic I, Dragne C, Filip M, et al. Subcyclo laser procedure results in patients with glaucoma. Romanian J Ophthalmol. 2018:62(4):296-299. doi:10.22336/rjo.2018.45 